Available online at www.iponlinejournal.com

\title{
Comparison of three dimensional accuracy of two implant level impression techniques; using three different elastomeric impression materials-an in vitro study
}

\author{
Jagadeesh Naik $^{1}$, Sidhartha S. P. Behera ${ }^{2 *}$, Girish Galagali ${ }^{3}$, Prakash Nidawani ${ }^{4}$ \\ ${ }^{1}$ Private Practitioner, ${ }^{2}$ Associate Professor, ${ }^{3}$ Professor and HOD, ${ }^{4}$ Professor, Dept. of Prosthodontics, ${ }^{2}$ Kims Dental College, Amalapuram, \\ Andhra Pradesh, ${ }^{\mathbf{3 , 4}}$ Navodaya Dental College and Hospital, Raichur, Karnataka, India
}

\begin{abstract}
Aim: To compare 3-Dimensional accuracy of open and closed impression technique using three elastomeric impression materials to transfer the intraoral position of implant fixture to the working cast.

Objective: To compare 3-Dimensional accuracy of open and closed tray impression technique by using addition silicone, condensation silicone and polyether impression materials for making implant level impressions.

Materials and Methods: Experiments was conducted to find the three dimensional accuracy of casts made with three impression materials for implant impressions by open and closed tray impression techniques. Five implant analogs were placed on a maxillary acrylic model; impression posts were screwed to implant analogs. 30 stock trays and 30 open window custom trays were prepared. These 30 stock trays and 30 open window custom trays were again divided into three subgroups by using three impression materials. Then the impressions were poured using die stone according to manufacturer's instructions. Specific dimensions of resultant cast was measured. The dimensional changes were measured using White light laser scanner and data analysis was done by XOV Rapidform Software.

Comparison between materials was done by ANOVA test followed by Tukey'smultiple comparison test. Comparison between two techniques was done by unpaired ' $t$ ' test.

Results: The data was subjected to statistical analysis which was done by ANOVA test followed by Tukey's multiple comparison test and Comparison between two techniques was done by unpaired' $t$ ' test. Statistical analysis shows significantly less difference in open tray technique when compared to closed tray impression technique.

Conclusion: Within the limitation of this study;1. Open tray impression technique is more accurate than the closed tray impression technique for making implant impression; 2. Polyether and Addition silicone elastomeric impression materials were there commended materials for implant impressions.
\end{abstract}

Keywords: Implants, Impression Techniques

\section{Introduction}

Reproducing the intraoral relationship of implants on working cast through impression procedures is the first step in achieving an accurate, passively fittingprosthesis. ${ }^{1}$ Consequently, recording the intraoral three-dimensional position of implants is a more critical task in the realization of implant supported than in tooth supported prostheses to ensure an accurate relationship transferred on the master cast. However, the results are not always consistent, and various studies reported greater accuracy with different impression techniques as well as impression materials. ${ }^{2}$ In dental implant prosthesis, fabrication of passively fitting prosthesis will lead to the long term success of the restorations. The critical aspect is to record the 3-dimensional orientation of the implant as it is present intra orally, other than reproducing fine surface detail for successful implant prosthodontics treatment. ${ }^{3}$ Implant-level impression making permits selection of the most appropriateabutments in the laboratory with abutment selection kits, which is helpful for situations where vertical space and/or angulation of the abutment are difficult to determine intra orally. In addition, it facilitates replacement of the healing caps by eliminating the need to cover the abutments with temporary restorations or protective caps. In particular, when the restoration connects directly to the implants, as in cases of insufficient vertical space, the definitive cast is obtained by an implant-level impression. ${ }^{4}$

\section{Materials and Methods Materials}

A edentulous maxillary acrylic model had been fabricated and five implant analogs of ADIN implants with impression posts were placed on the acrylic model, Maxillary perforated metal stock tray is used for making closed tray impression technique and Custom made maxillary acrylic tray for open tray impression technique. Three elastomeric impression materials such as Addition silicone impression material-Coltene, Condensation silicone impression material-Stomaflex and Polyether impression material-3M ESPE had been used.

\footnotetext{
*Corresponding Author: Sidhartha S. P. Behera, Dept. of Prosthodontics, Kims Dental College, Amalapuram, Andhra Pradesh, India Email: drsidprosthokims@gmail.com

http://doi.org/10.18231/j.aprd.2019.016
} 


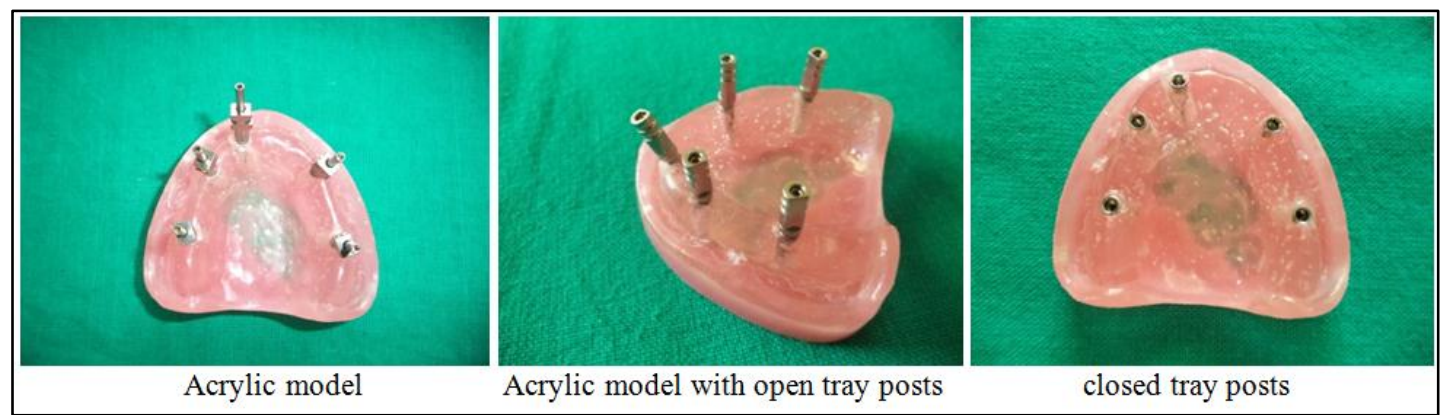

Fig. 1:

\section{Method of collection of the data}

Five implant analogs were placed on a maxillary acrylic model, impression posts were screwed to implant analogs. 30 stock trays and 30 open window custom trays were prepared. These 30 stock trays and 30 open window custom trays were again divided in to three subgroups by using three impression materials.

Group A(Open tray techniqe)

A)Polyether 10 impressions B]Condensation silicone 10 impressions C]Addition silicone 10 impressions.

Group A (Closed tray techniqe)

A)Polyether 10 impressions B]Condensation silicone 10 impressions C]Addition silicone 10 impressions.

\section{Impression making}

For the open tray technique, warmed boxing wax were adapted over the openings before loading the tray with impression material. After the tray was seated, the base plate wax was used to prevent flowing of the impression material through the openings and also to help identify the location of the impression copings. The copings were loosened with a driver and removed; the tray was separated from the master cast while the impression copings remained locked in the impression. The guide pins were placed back into the open tray impression copings from the top, while an implant analog was connected to the hex and the guide pins were hand tightened.

For the open tray technique copings remained on the master cast after the impression material had polymerized when the tray was removed. These impression copings were removed one at a time from the resin model and attached to an implant analog. The combined impression copinganalog unit was inserted into the impression by firmly pushing it into place to full depth. Type IV gypsum product.(Die stone) -Kalabhai was mixed with water according to manufacturer recommendation (16 $\mathrm{ml}$ water will be used with each $70 \mathrm{gm}$ powder). The stone was mixed and was poured on a vibrator (Whip Mix Corporation, Louisville, KY). Casts were separated from the impressions after allowing the stone to set for 1 hour, followed by trimming and labeling to prepare for measurements.

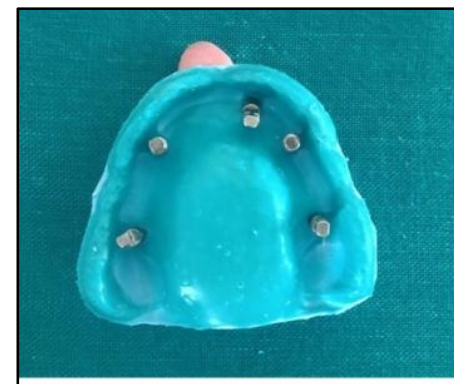

Open tray impression

Fig. 2:

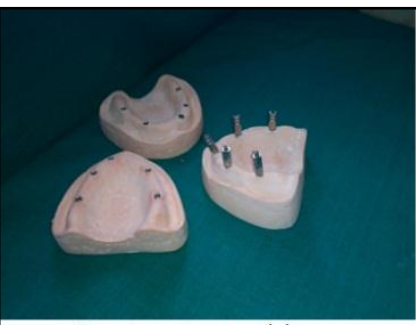

Open tray stone models

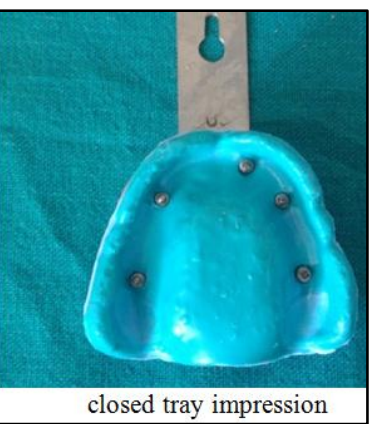

Fig. 3:

\section{Measurement protocol}

\section{Dimensional measurements}

The dimensional changes were measured using White light laser scanner and

data analysis was done by XOV Rapidform Software.

\section{Statistical analysis}

Descriptive statistics such as mean and SD was used. Comparison between materials was done by ANOVA test followed by Tukey's multiple comparison test. Comparison between two techniques was done by unpaired ' $t$ ' test. A pvalue less than 0.05 were considered as significant.



Fig. 4: 


\section{Results}

Table1:Distance comparison open tray vs close tray; Addition silicone

\begin{tabular}{|c|c|c|c|c|c|c|}
\hline \multicolumn{4}{|c|}{ Closed trayimpression technique } & \multicolumn{3}{c|}{ Open tray impression technique } \\
\hline $\begin{array}{c}\text { Reference } \\
\text { point }\end{array}$ & $\begin{array}{c}\text { Standard } \\
\text { model }\end{array}$ & Addition silicone & Mean diff. & $\begin{array}{c}\text { Standard } \\
\text { model }\end{array}$ & $\begin{array}{c}\text { Addition } \\
\text { silicone }\end{array}$ & Mean diff. \\
\hline A-B & $18.394 \pm 0.0$ & $18.377 \pm 0.04$ & 0.017 & $18.005 \pm 0.0$ & $18.005 \pm 0.004$ & 0.00 \\
\hline B-C & $15.533 \pm 0.0$ & $15.531 \pm 0.006$ & 0.002 & $17.247 \pm 0.0$ & $17.247 \pm 0.006$ & 0.00 \\
\hline C-D & $25.62 \pm 0.0$ & $25.620 \pm 0.003$ & 0.00 & $27.728 \pm 0.0$ & $27.728 \pm 0.02$ & 0.00 \\
\hline D-E & $20.299 \pm 0.0$ & $20.299 \pm 0.005$ & 0.00 & $20.145 \pm 0.0$ & $20.145 \pm 0.01$ & 0.00 \\
\hline
\end{tabular}

Table2:Distance comparison open tray vsclose tray;Condensation silicone

\begin{tabular}{|c|c|c|c|c|c|c|}
\hline \multicolumn{4}{|c|}{ Closed trayimpression technique } & \multicolumn{3}{c|}{ Open trayimpression technique } \\
\hline $\begin{array}{c}\text { Reference } \\
\text { point }\end{array}$ & $\begin{array}{c}\text { Standard } \\
\text { model }\end{array}$ & $\begin{array}{c}\text { Condensation } \\
\text { silicone }\end{array}$ & $\begin{array}{c}\text { Mean } \\
\text { diff. }\end{array}$ & $\begin{array}{c}\text { Standard } \\
\text { model }\end{array}$ & $\begin{array}{c}\text { Condensation } \\
\text { silicone }\end{array}$ & Mean diff. \\
\hline A-B & $18.394 \pm 0.0$ & $18.419 \pm 0.02$ & 0.025 & $18.005 \pm 0.0$ & $18.022 \pm 0.03$ & 0.017 \\
\hline B-C & $15.533 \pm 0.0$ & $15.543 \pm 0.01$ & 0.01 & $17.247 \pm 0.0$ & $17.252 \pm 0.04$ & 0.005 \\
\hline C-D & $25.62 \pm 0.0$ & $25.625 \pm 0.006$ & 0.005 & $27.728 \pm 0.0$ & $27.743 \pm 0.01$ & 0.015 \\
\hline D-E & $20.299 \pm 0.0$ & $20.310 \pm 0.02$ & 0.011 & $20.145 \pm 0.0$ & $20.155 \pm 0.009$ & 0.01 \\
\hline
\end{tabular}

Table5: Distance comparison open tray vs close tray;Polyether

\begin{tabular}{|c|c|c|c|c|c|c|}
\hline \multicolumn{4}{|c|}{ Closed trayimpression technique } & \multicolumn{3}{c|}{ Open trayimpression technique } \\
\hline $\begin{array}{c}\text { Reference } \\
\text { point }\end{array}$ & $\begin{array}{c}\text { Standard } \\
\text { model }\end{array}$ & Polyether & Mean diff. & $\begin{array}{c}\text { Standard } \\
\text { model }\end{array}$ & Polyether & $\begin{array}{c}\text { Mean } \\
\text { diff. }\end{array}$ \\
\hline A-B & $18.394 \pm 0.0$ & $18.392 \pm 0.01$ & 0.002 & $18.005 \pm 0.0$ & $18.005 \pm 0.01$ & 0.00 \\
\hline B-C & $15.533 \pm 0.0$ & $15.532 \pm 0.01$ & 0.001 & $17.247 \pm 0.0$ & $17.247 \pm 0.003$ & 0.00 \\
\hline C-D & $25.62 \pm 0.0$ & $25.619 \pm 0.006$ & 0.001 & $27.728 \pm 0.0$ & $27.728 \pm 0.009$ & 0.00 \\
\hline D-E & $20.299 \pm 0.0$ & $20.299 \pm 0.006$ & 0.00 & $20.145 \pm 0.0$ & $20.145 \pm 0.009$ & 0.00 \\
\hline
\end{tabular}

Statistical analysis shows significantly less difference in open tray technique when compared to closed tray impression technique. Based on the data open tray technique is more accurate than closed tray technique for making implant impression.

\section{Discussion}

In dental implant prosthesis, fabrication of passively fitting prosthesis will lead to the long term success of the restorations. Reproducing the intraoral relationship of implants through impression procedures is the first step in achieving an accurate, passively fitting prosthesis. The critical aspect is to record the 3-dimensional orientation of the implant as it is present intra orally, other than reproducing fine surface detail for successful implant prosthodontics treatment.

Previous studies compared the accuracy of pick-up on transfer impressiontechniques, ${ }^{5-12}$ and few studies showed more accurate impressions with the transfertechnique. ${ }^{13}$ Daoudi et al investigated repositioning of the copings after making the transfer impression by 3 different groups of people: senior dentists, post graduate dental students, and dental technicians. The copings never returned to the original position and this was believed to be the primary source of error in the transfer impression technique. This error could be multiplied when the impression is made in situations of multiple implant placement. It was found that for situations in which there were 4 or more implants, more studies showed more accurate impressions with the pick-up technique than the transfer technique. Various impression materials were tested, such as condensation silicone, polysulfide, reversible hydrocolloid, irreversible hydrocolloid, and plaster, but polyether and addition silicone were used most frequently. The studies comparing the accuracy of polyether and addition silicone, ${ }^{9-14}$ and few studies reported that the accuracy did notdiffer. ${ }^{15,16}$ Lee et alreported that putty and light-body combination addition silicone impression material was more accurate than medium-body polyether impression material when the implant was placed deep subgingivally. The findings of this in vitro study do not support rejection of the null hypothesis, as Statistical analysis shows significantly less difference in open tray technique when compared to closed tray impression technique. ${ }^{17}$ Based on the data open tray technique is more accurate than closed tray technique for making implant impression with polyether and addition silicon elastomeric material showing the higher accuracy of Implant level Impressions.

\section{Conclusion}

Within the limitation of this study

1. Open tray impression technique is more accurate than the closed tray impression technique for making implant impression 
2. Polyether and Addition silicone elastomeric impression materials were the recommended materials for implant impressions.

It is important to understand all of the prosthetic steps necessary for the treatment success. With proper selection of impression material and technique to make implant level impression are more predictable when compared with conventional techniques. Future studies can be taken up with various other material lsand techniques subjected to the more scientific evaluation under simulated clinical conditions.

\section{Source of Funding}

None.

\section{Conflict of Interest}

None.

\section{Reference}

1. Steigenga JT, Al-Shammari KF, NocitiFH, Misch CE, Wang H.Dental implant designand its relationship to long-term implant success. Implant Dent 2003;12(4):306-17.

2. The glossary of prosthodontic terms July. J Prosthet Dent2005;94(1):10-92.

3. S.Reddy,KPrasad,HVakil, A Jain,RChowdary. Accuracy of impressions with different impression materials in angulated implants.Nigerian J ClinPract2013;16(3).

4. Choi JH, Lim YJ, Yim SH, Kim CW. Evaluation of the accuracy of implant-levelimpression techniques for internalconnection implant prostheses in parallel anddivergent models.Int J Oral Maxillofac Implants 2007;22:761-68.

5. Barrett MG, de Rijk WG, Burgess JO. The accuracy of six impression techniques forosseointegrated implants. $J$ Prosthod 1993;2:75-82.

6. Del'Acqua MA, Arioli-Filho JN, Compagnoni MA, MolloFd A Jr. Accuracy ofimpression and pouring techniques for an implant-supported prosthesis. Int J OralMaxillofac Implants 2008;23:226-36

7. DaoudiMF, Setchell DJ, Searson LJ. An evaluation of three implant level impressiontechniques for single tooth implant.Eur J ProsthodontRestor Dent 2004;12:9-14.
8. De La Cruz JE, Funkenbusch PD, Ercoli C, Moss ME, Graser GN, Tallents RH et al.Verification jig for implant-supported prostheses: A comparison of standard impressionswith verification jigs made of different materials. $J$ Prosthet Dent 2002;88:329-36.

9. Carr AB. Comparison of impression techniques for a fiveimplant mandibular model.Int J Oral Maxillofac Implants 1991;6:448-55.

10. Carr AB. Comparison of impression techniques for a twoimplant 15-degree divergentmodel.Int J Oral Maxillofac Implants 1992;7:468-75.

11. Conrad HJ, Pesun IJ, DeLong R, Hodges JS. Accuracy of two impression techniqueswith angulated implants. J Prosthet Dent 2007;97:349-56.

12. Wenz HJ, Hertrampf K. Accuracy of impressions and casts using different implantimpression techniques in a multiimplant system with an internal hex connection. IntJOral Maxillofac Implants 2008;23:39-47.

13. Humphries RM, Yaman P, Bloem TJ. The accuracy of implant master castsconstructed from transfer impressions. Int J Oral Maxillofac Implants 1990;5:331-6.

14. LorenzoniM, Pertl C, Penkner K, PolanskyR, Sedaj B, Wegscheider WA.Comparisonof the transfer precision of three differentimpression materials incombination with transfer caps for the Frialit-2 system. J Oral Rehabil 2000;27:629-38.

15. Wenz HJ, HertrampfK . Accuracy of impressions and casts using different implantimpression techniques in a multiimplant system with an internal hex connection. Int JOral Maxillofac Implants 2008;23: 39-47.

16. Daoudi MF, Setchell DJ, Searson LJ. A laboratory investigation of the accuracy oftwo impression techniques for single-tooth implants.Int J Prosthodont2001;14:152-8.

17. Lee H,Ercoli C, Funkenbusch PD, Feng C. Effect of subgingival depth of implantplacement on the dimensional accuracy of the implant impression: an in vitro study. JProsthet Dent 2008;99:107-13.

How to cite this article: Naik J, Behera SSP, Galagali G, Nidawani P. Comparison of three dimensional accuracy of two implant level impression techniques; using three different elastomeric impression materials-an in vitro study. Ann Prosthodont Restor Dent 2019;5(3):68-71. 